\title{
RESEARCH ON INFLUENCE OF MEMBER INITIAL CURVATURE ON STABILITY OF SINGLE-LAYER SPHERICAL RETICULATED DOMES
}

\author{
Yang Ding ${ }^{1,2}$ and Tian-long Zhang ${ }^{1, *}$ \\ ${ }^{1}$ School of Civil Engineering, Tianjin University, Tianjin, China \\ ${ }^{2}$ Key Laboratory of Coast Civil Structural Safety of the Ministry of Education, Tianjin University, Tianjin, China \\ *(Corresponding author: E-mail: ztl1019@126.com)
}

\section{A B S T R A C T}

The distribution modes and magnitudes of member initial curvature significantly influence the stability of single-layer reticulated dome. Based on the deflection curvature of a compression bar with unequal end moments, a numerical simulation method for member initial curvature considering end moment effects is proposed using multi-beam methods based on ABAQUS. The approach is verified through a comparison of experiments using different systems. Using Kiewitt-8 dome as an example, the extent of the member initial curvature's effect on the stability bearing capacity of these dome for different structure parameters is studied. The results show that the bending moments at the bar's ends can affect the deflection shape of a compression bar and that the most unfavorable condition occurs when the member initial curvatures are coordinate with the deflection shapes. The reduction degree of the stability load-bearing capacity of such dome modelled using member initial curvatures considering end moments is greater than that for curvatures without considering end moments. The influence of different member initial curvature amplitudes on the stability of these dome varies significantly, and a reasonable maximum value of the member initial curvature is proposed for performing stability analysis based on the structure response and realistic construction conditions.
A R T I C L E H I S T O R Y

$\begin{array}{ll}\text { Received: } & \text { 13 February } 2017 \\ \text { Revised: } & \text { 16 December } 2017 \\ \text { Accepted: } & \text { 17 December } 2017\end{array}$

\section{K E Y W O R D S}

Single-layer spherical reticulated dome;

Nonlinear stability;

Bearing capacity;

Member Initial curvature;

End Moment

\section{Introduction}

The single-layer reticulated dome is a structure formed with slender compression members and rigid joints. Traditional design procedures for longspan space reticulated structures assume that the shape of each member is a straight line and that the stability analysis of reticulated structures considering the effect of geometric nodal imperfections is conducted without accounting for the effects of member initial defects under normal conditions [1,2]. As a principal factor, the range of geometric nodal imperfections is required and controlled by structural construction codes and technical specifications $[3,4]$. However, prefabricated bars of these dome may unavoidably become bent during production and transport, resulting in member initial curvatures. The member initial curvatures may reduce the buckling strength of the bars under axial compression load, due to the $P-\delta$ effect, leading to a redistribution of forces among members in the neighboring regions and to an interaction between individual member buckling and global instability of the dome, which may adversely affect the stability bearing capacity of the dome $[5,6]$

To investigate the influence of the $P-\delta$ effect on the stability bearing capacity, adopting shell element to model thin-walled members based on the finite element (FE) method is a direct approach. Kumar et al. [7] and Lotfollahi et al. [8] proposed shell elements in FE models to investigate the post-buckling performance of steel braces. However, it is extremely complicated to model and analyze a single-layer reticulated dome using shell elements due to the demanding computational requirements. Another possible solution is using beam elements. A plastic hinge model was developed by Liew et al. [9] based on the buckling of members without physically altering the member geometry, although this approach is not practical for simulating the cyclic hysteresis behaviour of struts due to the lack of unloading curves. Qi et al. [10] introduced a phenomenological model, the Marshall model [11], to consider this interaction. However, the applicable strain range is too narrow to simulate the large deformation of the strut. A theoretical strut model was proposed in Reference [12] using the one-element-per-member method to account for the interaction between individual member buckling and global structural instability, which requires an assumption for the effective length first. Chan et $a l$. [13] presented a single-imperfect-element-per-member method that need not assume the effective length and that includes the effects of initial imperfections in the element stiffness. However, this method is based on the underlying assumption that the element is prismatic and elastic.

When a design load induces plasticity in a single-layer spherical reticulated dome, the multi-element-per-member method can accurately simulate the plastic non-linear behavior by dividing one member into more straight elements to study second-order effects on the non-linear stability of the dome. The multielement-per-member method can present the $P$ - $\delta$ effect under different endconstraint conditions and avoid the limitations of the effective length assumption. Plastic hinges at different locations in each member can be captured by the multi-beam method rather than the one-element method [14,15]. Zhou et al. [16] proposed initial bending 3D link elements and initial bending 3D beam elements assuming that the initial bending shape of the members is a half-period sine curve, and studied the effects of the bearing capacity of the cable-arch structures. Yan et al. [17] adopted the multi-beam method to model the initial curvature of members based on FE analysis and investigated the influence of the direction angle and the amplitude of random variables of the initial curvature of members on the stability of a reticulated shell structure. Wang et al. [18] established multi-beam elements with an initial curvature by combining a halfperiod sine curve and a full-period sine curve to determine the effects on the buckling capacity and seismic response. In the Hong Kong Steel Code [19,20], a second-order analysis method uses a length-free assumption by introducing a half-period sine curve as the member initial curvature. However, this memberonly initial bow function is too simple to consider the end moment effect.

In practice, the joints of single-layer reticulated dome are nearly rigid or semi-rigid, and the axial forces as well as the bending moments are transmitted directly by the joints. The bending moments at the bars' ends may affect the buckling shapes of compression bars in single-layer reticulated dome. The existing functions of member initial curvatures do not consider the end moment effect. In this paper, the deflection equilibrium equation for a compression bar with end moments is derived; the results indicate that member initial curvature with a half-period sine curve is not the most unfavorable condition. According to the deflection equilibrium equations, two types of member initial curvatures are proposed that consider the end moment effect to avoid the limitations of the existing curvature functions. A numerical modelling method for determining the member initial curvature in single-layer reticulated dome is proposed via the multi-beam method based on the ABAQUS finite-element program. In addition, the approach is verified through comparison with experiments on different systems. Taking Kiewitt- 8 dome as an example, the extent of the member initial curvature's influence on the stability bearing capacity of single-layer reticulated dome with different structure parameters is studied. The influence of different member initial curvature amplitudes is discussed to inform the selection of a reasonable maximum value of the member initial curvature for stability analysis of single-layer reticulated dome.

\section{Member initial curvature of compression bar}

\subsection{Member initial curvature of compression bar with hinged joints}

A compression bar with pinned supports balances against bending deformation as soon as it reaches a critical equilibrium state under an axial compression load, as shown in Fig. 1. 


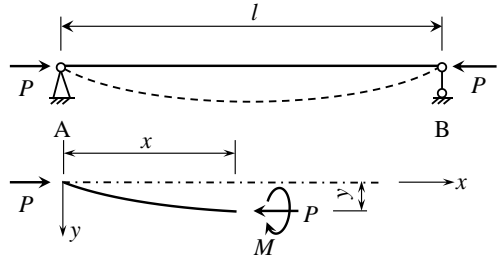

Fig. 1 Deflection shape of compression bar with hinged joints

Given distance $x$ from the left end, the force equilibrium equation and approximate deflection differential equation of the bar are specified as

$$
E I y^{\prime \prime}=M=-P y
$$

where $l$ is the length of a single bar or member; $E$ is the elastic modulus of material; $I$ is the inertia moment of bar section; $y$ is the deflection of a node in the local normal direction; $M$ is the bending moment of bar section; $P$ is the stability load-bearing capacity of compression bar. Let $k^{2}=P / E I$ such that Eq. 1 becomes

$y^{\prime \prime}+k^{2} y=0$

The general solution of Eq. 2 is obtained as

$$
y=C_{1} \operatorname{sinkx}+C_{2} \operatorname{sinkx}
$$

where $C_{1}$ and $C_{2}$ are coefficients. Eq. 3 is solved by substituting the boundary conditions of the compression bar at the two ends: $y(0)=0, y(x)=0$. The result is $C_{1} \sin k l=0$, where $k l=n \pi(n= \pm 1, \pm 2 \ldots)$. Therefore, the deflection curve of a compression bar with hinged joints under the most unfavorable condition when $n=1$ is obtained as follows

$y=C_{1} \sin (\pi x / l)$

Eq. 4 accounts for the fact that the deflection shape of the compression bar with hinged joints is a half-period sine curve, which is the most unfavorable buckling mode. Therefore, the lowest stability bearing capacity is obtained when taking a half-period sine curve as the member initial curvature of a compression bar with pinned supports. The member initial curvature of a compression bar with hinged joints is specified as

$$
\delta=\delta_{\max } \sin (\pi x / l)
$$

where $\delta$ is the node imperfection considering the member initial curvature in the local normal direction; $\delta_{\max }$ is the amplitude of a member initial curvature imperfection.

\subsection{Member initial curvatures of compression bar with end moments}

When simulating the initial bending of members, the effect of end moments transmitted by rigid joints or semi-rigid joints should be considered in long-span space structures. The deformation shape of a compression bar under unequal bending moments at the two ends, $M_{\mathrm{A}}$ and $M_{\mathrm{B}}$, is shown in Fig. 2 .

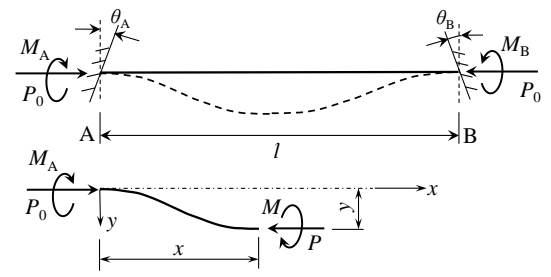

Fig. 2 Deflection shape of compression bar with end moments

$M_{A}$ and $M_{B}$ are positive if the synclastic curvature deformation is generated by $M_{A}$ and $M_{B}$. Otherwise, the coefficient with the larger absolute value is positive and the other coefficient is negative if the anticlastic curvature deformation is generated by $M_{A}$ and $M_{B}$. The deflection differential equation of a compression bar under unequal bending moments at the two ends is as follows

$$
y^{\prime \prime}+k^{2} y=\frac{M_{\mathrm{A}}+M_{B}}{E I l} x-\frac{M_{\mathrm{A}}}{E I}
$$

where $k^{2}=P_{0} / E I$. The general solution of Eq. 6 is

$y=C_{1} \sin k x+C_{2} \cos k x+\frac{M_{\mathrm{A}}+M_{B}}{k^{2} E I l} x-\frac{M_{\mathrm{A}}}{k^{2} E I}$

where $C_{1}$ and $C_{2}$ are coefficients. Eq. 7 is solved by substituting the boundary conditions of the compression bar at the two ends: $y(0)=0, y(x)=0$. The coefficients of Eq. 7 are

$C_{1}=-\frac{M_{\mathrm{A}} \cos k l+M_{\mathrm{B}}}{k^{2} E I \sin k l}$

$C_{2}=\frac{M_{\mathrm{A}}}{k^{2} E I}$

The deflection curve result of Eq. 7 for a compression bar under unequal bending moments at two ends when substituting Eq. 8 and Eq. 9 into the equation is

$y=-\frac{M_{\mathrm{A}} \cos k l+M_{\mathrm{B}}}{k^{2} E I \sin k l} \sin k x+\frac{M_{\mathrm{A}}}{k^{2} E I} \cos k x+\frac{M_{\mathrm{A}}+M_{B}}{k^{2} E I l} x-\frac{M_{\mathrm{A}}}{k^{2} E I}$

Eq. 10 shows that the deformation curve of compression members under unequal bending moments is the combination of a cosine curve and a sine curve, which are related to both the values and directions of the bending moments bearing at the bar's ends. Because the most unfavourable situation is that in which the initial bending of the bar is in accord with its deformation shape, the member initial curvature should be determined by the distribution of moments at both ends.

Through the foregoing analysis, an advanced method for determining the member initial curvature is presented considering the distribution of moments at the bar's ends. First, a nonlinear full-range analysis of structures without any member defects is performed to obtain the moment distributions of all member ends in the critical state. Then, different initial bending functions are selected to modify the corresponding coordinates of the internal nodes in each member. To simplify the calculation, two types of member initial curvatures are established based on the different signs of the end moments, as shown in Fig. 3.

The first type of member initial curvatures (MIC 1st) considers that the moments at both ends are positive when the member deformation is a synclastic curvature shape. To simplify the analysis, it is assumed that the member deflection equation is a half-period sinusoid curve similar to the initial bending function, as shown in Fig.3 (a). Hence, the initial bending amplitude of the middle of the member is considered to have a maximum value $\delta$; meanwhile, the initial bending direction should agree with the deflation direction of the compression bar. The MIC 1st is specified as

$\delta=\delta_{\max } \sin (\pi x / l)$

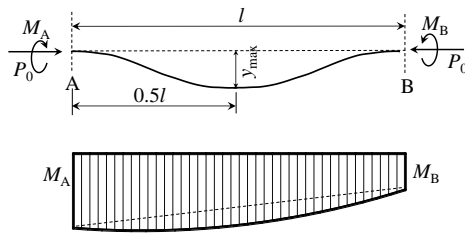

(a) Identical moment signs

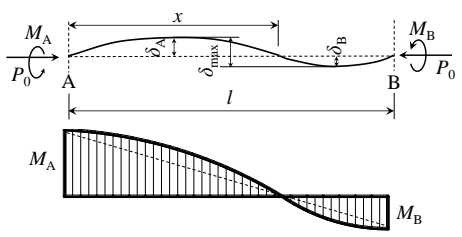

(b) Opposite moment signs

Fig. 3 Two types of member initial curvatures based on end moments 
The second type of member initial curvature (MIC 2nd) considers that the bar deformation takes on an anticlastic curvature shape with moments of different signs at the bar's two ends. To simplify the analysis, it is assumed that the member deformation shape consists of two half-period sinusoid curves in opposite directions, as shown in Fig. 3(b), and that the deformation value of the point where the moment equals zero is zero as well. Simultaneously, the maximum value $\delta$ of the initial bending amplitude is specified at the middle of the half-period sinusoid curve, where the end moment's absolute value is greater. MIC 2nd is specified as

$$
\delta=\left\{\begin{array}{cc}
\delta_{\max }\left(\frac{M_{\mathrm{A}}}{M_{\mathrm{A}}-M_{\mathrm{B}}}\right) \sin \left[\frac{\pi x\left(M_{\mathrm{A}}-M_{\mathrm{B}}\right)}{M_{\mathrm{A}} l}\right] & , x \leq \frac{M_{\mathrm{A}}}{M_{\mathrm{A}}-M_{\mathrm{B}}} l \\
-\delta_{\max }\left(\frac{M_{B}}{M_{\mathrm{A}}-M_{\mathrm{B}}}\right) \sin \frac{\pi\left(x-\frac{M_{\mathrm{A}}}{M_{\mathrm{A}}-M_{\mathrm{B}}}\right)\left(M_{\mathrm{A}}-M_{\mathrm{B}}\right)}{-M_{\mathrm{B}} l} & , x>\frac{M_{\mathrm{A}}}{M_{\mathrm{A}}-M_{\mathrm{B}}} l
\end{array}\right.
$$

Eq. 12 shows that the amplitude of the initial bending equals zero at the point $x=M_{A} l /\left(M_{A}-M_{B}\right)$, and the maximum value $\delta$ of the initial bending amplitude is obtained at the point $x=M_{A} l /\left(2 M_{A}-M_{B}\right)$, where $M_{A}>M_{B}$. The direction of this part of the half-period sinusoid curves, where $x<M_{A} l /\left(M_{A}-\right.$ $M_{B}$ ), should agree with the deflation direction of the compression bar. The amplitude of the initial bending is $\left(-M_{B} \delta / M_{A}\right)$ at the point $x=\left(M_{A}+0.5 M_{B}\right) l /$ $\left(M_{A}-M_{B}\right)$ in the other part of the half-period sinusoid curves, the direction of which is opposite to the direction at the point $x=M_{A} l /\left(2 M_{A}-2 M_{B}\right)$.

The modelling of steel space structures is conducted using the ABAQUS finite-element program, and beam B31 elements, 3D linear finite strain beam elements based on Timoshenko beam theory, are selected to simulate the members of the structures. Fig. 4 shows that each bar is meshed with 8 B31 elements using the multi-beam method.
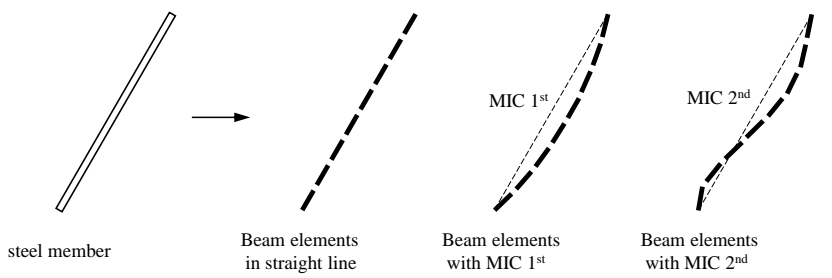

Fig. 4 Meshing rules of bars

The process of establishing structure models with member initial curvature considering the moments at the bars' ends is shown in Fig. 5. The modelling process is divided into end moment analysis and MIC computation. First, we extract the value and the direction of each bar's end moments based on the stability analysis results of the original structural model with straight members. Second, we select the MIC function and calculate the parameters according to Eq. 11 and Eq. 12. Finally, we modify the internal nodes coordinates of each bar to revise the structural model. All of the results are extracted and computed using the MATLAB program.

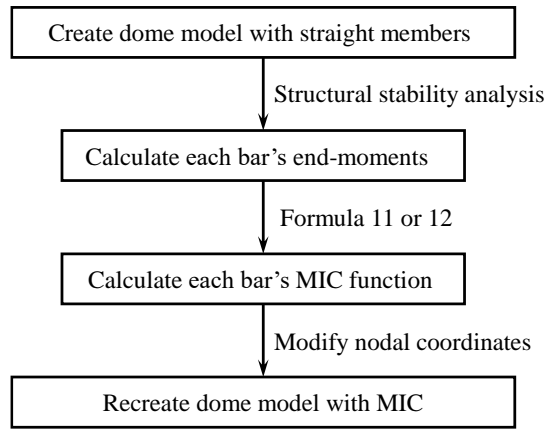

Fig. 5 Flow chart of structure modelling with MIC

\section{Model verification}

\subsection{Single-bar system}

To verify the method for establishing a structure model with member initial curvature considering end moments, the analysis single-bar experiment conducted by Sherman with both ends fixed under axial compression, relative to Reference [21], is analyzed. The single bar is made of steel circular pipes and measures $5.72 \mathrm{~m}$ in length, $114 \mathrm{~mm}$ in outer diameter and $2.3 \mathrm{~mm}$ in thickness, as shown in Fig. 6.

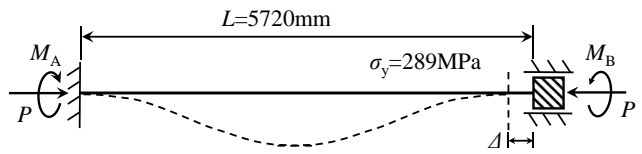

Fig. 6 Sherman's experiment on steel pipe under axial load

Two types of member initial curvatures are chosen to simulate the fullrange compressive buckling process under Sherman's test. The maximum value of the initial bending amplitude $\delta_{\max }$ is specified as $l / 500$, where $l$ is the length of the bar and $\sigma_{\mathrm{y}}$ is the yield strength of steel. The load factor of the stability load-bearing capacity of the model considering different member initial curvatures is specified as

$C_{0}=P / P_{0}$

where $P$ is the stability load-bearing capacity of the model without any member initial defects and $P_{0}$ is the stability load-bearing capacity of the model established using member initial curvatures. The load-displacement curves and load-bearing capacity factor $C_{0}$ of the system using different MIC functions are shown in Fig. 7.

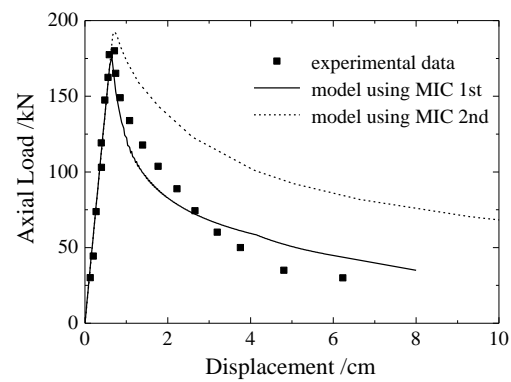

(a) Load-displacement curves

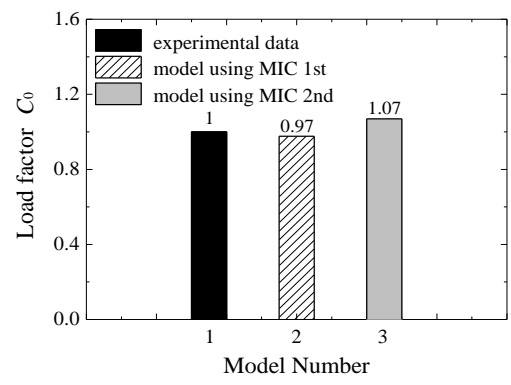

(b) Load-bearing capacity factors

Fig. 7 Model results of Sherman's experiment

Fig. 7 shows that the load-displacement curve of the model established using the first type of member initial curvature is closer to the test result. The compressive bearing capacities of the test sample, MIC 1st and MIC 2nd are $180.4,176.1$, and $192.8 \mathrm{kN}$, respectively. These results are obtained because the signs of the moments at the two ends are the same in the single-bar experiment. The first type of initial bending function agrees better with the test case; thus, the compressive bearing capacity is the lowest, proving that the most unfavourable condition occurs when the signs of the end moments are the same.

\subsection{Double-bar system}

To verify the method for establishing a structure model with member initial curvature considering end moments, a numerical simulation of the double-bar experiment with fixed ends under vertical load reported by Shen in Reference [22] is conducted. The double-bar system is composed of steel circular pipes with strong nonlinear mechanical properties, as shown in Fig. 8. The external diameter and thickness of the pipe sections are $114 \mathrm{~mm}$ and $4 \mathrm{~mm}$, and two types of member initial curvatures are chosen to compare the full-range 
compressive buckling process of the system without member initial curvature. The maximum value of the member initial curvature amplitude $\delta_{\max }$ is specified as $l / 500$, where $l$ is the length of each bar; $E$ is the elastic modulus of steel; $\sigma_{\mathrm{y}}$ is the yield strength of steel. To compare the influence of semi-rigid joints on end moments, the bending stiffness of rigid joints is reduced to produce a semi-rigid joint system. The load-displacement curves and the bearing capacity factor $C_{0}$ of the rigid system and the semi-rigid system using different MIC functions are shown in Fig. 9.

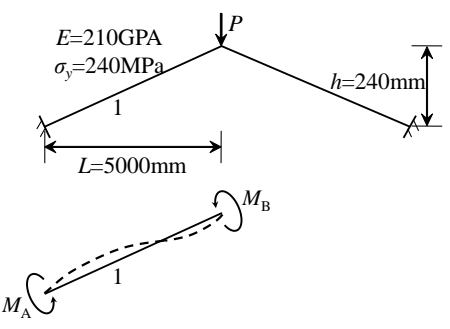

Fig. 8 Double-bar system under vertical load

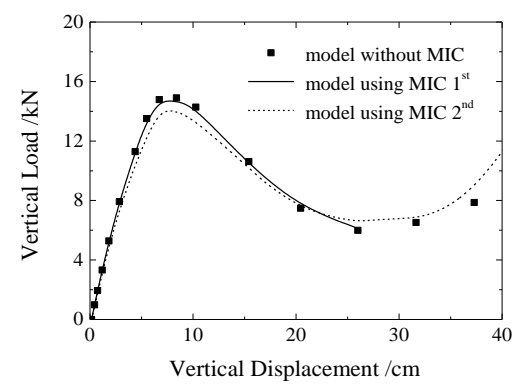

(a) Load-displacement curves with rigid joints

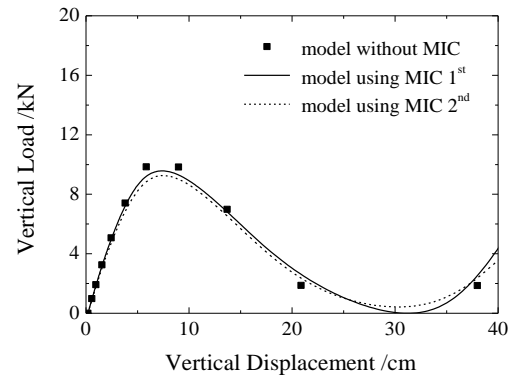

(b) Load-displacement curves with semi-rigid joints

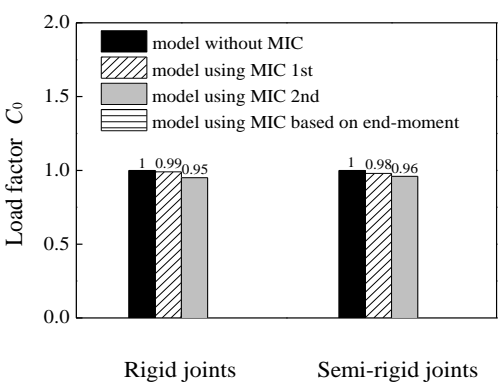

(c) Load-bearing capacity factor

Fig. 9 Model results for the double-bar system

Fig. 9 (a) shows that the stability bearing capacities of the three types of rigid joints models are $14.8,14.7$, and $14.1 \mathrm{kN}$, and the capacity of the model using the second type of member initial curvature is the lowest. Fig. 9 (b) shows that the stability bearing capacities of the three types of semi-rigid joints models are $9.84,9.63$, and $9.47 \mathrm{kN}$, and the capacity of the model using the second type of member initial curvature is also the lowest. These results are obtained because the signs of the moments at the bars' ends transmitted by rigid or semirigid joints are opposite in the critical state, and the deformation shape of each bar is the anticlastic curvature under vertical load, proving that the second type of member initial curvature represents the most unfavourable condition when the end moments are opposite.

\subsection{Hexagon frame system}

To verify the method for establishing a structure model with member initial curvature considering end moments, a numerical simulation of the hexagon frame system experiment with hinged supports under vertical load reported by Papadrakakisn in Reference [23] is conducted, as shown in Fig. 10. The six bars around the middle loading point are compression bars; two types of member initial curvature are chosen to compare the full-range compressive buckling process of the system without member initial curvature. The maximum value of the initial bending amplitude $\delta_{\max }$ is specified as $1 / 500$, where $l$ is the length of each bar; $I$ is the inertia moment of a pipe section; $J$ is the torsional constant of a circular section; $E$ is the elastic modulus of steel; $G$ is the shear modulus of steel; $\sigma_{\mathrm{y}}$ is the yield strength of steel. To compare the influence of semi-rigid joints on end moments, the bending stiffness of rigid joints is reduced to produce a semi-rigid joint system. The load-displacement curves and the bearing capacity factor $C_{0}$ of the rigid system and the semi-rigid system using different MIC functions are shown in Fig. 11.
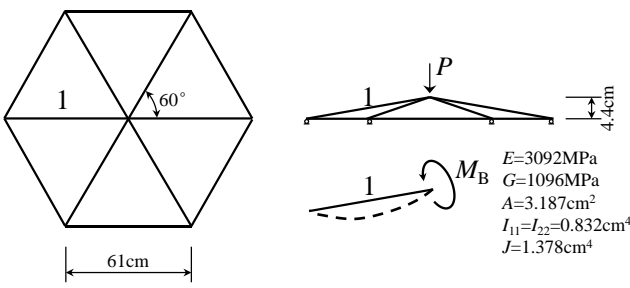

Fig. 10 Hexagon frame system under vertical load

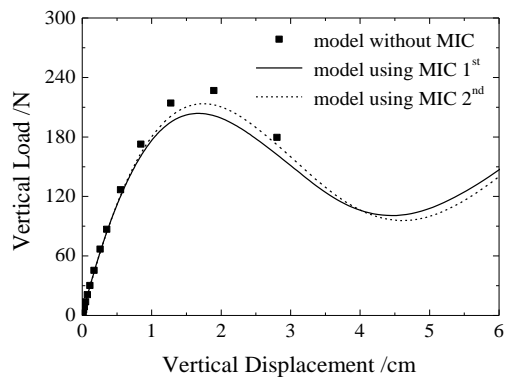

(a) Load-displacement curves with rigid joints

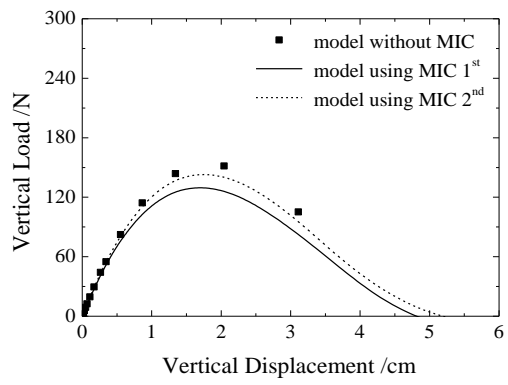

(b) Load-displacement curves with semi-rigid joints

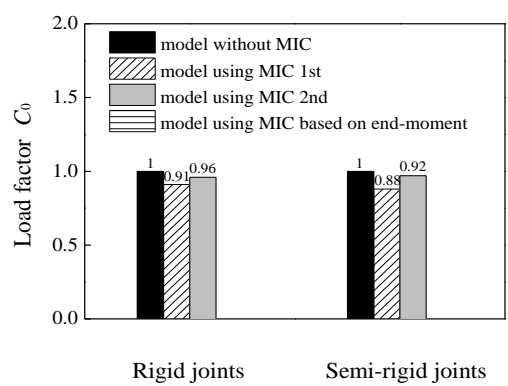

(c) Load-bearing capacity factor

Fig. 11 Model results for the hexagon frame system

Fig. 11 (a) shows that the stability bearing capacities of the three types of rigid joints models are 226.8, 206.6, and $218.3 \mathrm{~N}$, and the capacity of the model using the first type of member initial curvatures is the lowest. Fig. 11 (b) shows that the stability bearing capacities of the three types of semi-rigid joints models 
are $151.0,133.1$, and $146.0 \mathrm{~N}$, and the capacity of the model using the first type of member initial curvatures is also the lowest. These results are obtained because the moment of each compression bar's supporting end is zero. In this one-end hinged situation, the deformation shape of each compression bar presents synclastic curvature under load, proving that the first type of member initial curvature represents the most unfavourable condition when one endmoment is zero.

\subsection{Hexagon star dome system}

To verify the method for establishing a structure model with member initial curvature considering end moments, a numerical simulation of the hexagon star dome system experiment with hinged supports under vertical loads reported by Meek and Tan in Reference [24] is conducted, as shown in Fig. 12. Three types of dome system models considering different member initial curvatures are established for comparison with models with no member initial curvatures. The first type consists of all the member initial curvatures as function 12 (MIC 1st), the second type consists of all the member initial curvatures as function 13 (MIC 2nd), and for the third type, function 12 (MIC 1st) or function 13 (MIC 2nd) is chosen for each bar based on the bars' moments at both ends. The maximum value of the initial bending amplitude $\delta_{\max }$ is specified as $l / 500$, where $l$ is the length of the bar; $I$ is the inertia moment of a pipe section; $J$ is the torsional constant of a circular section; $E$ is the elastic modulus of steel; $G$ is the shear modulus of steel; $\sigma_{\mathrm{y}}$ is the yield strength of steel. To compare the influence of semi-rigid joints on end moments, the bending stiffness of rigid joints is reduced to produce a semi-rigid joint system. The load-displacement curves and the bearing capacity factor $C_{0}$ of the rigid system and the semi-rigid system using different MIC functions are shown in Fig. 13.

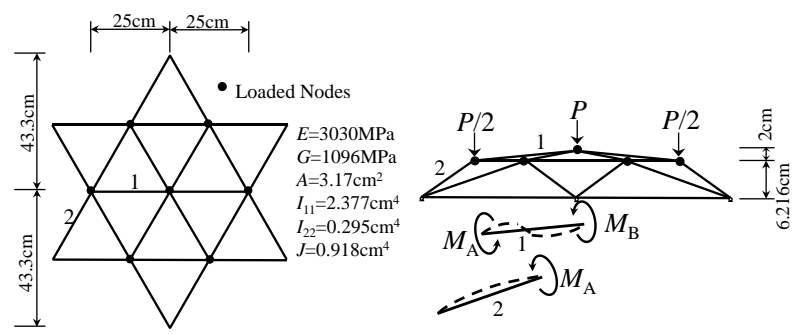

Fig. 12 Hexagon star dome system under vertical load

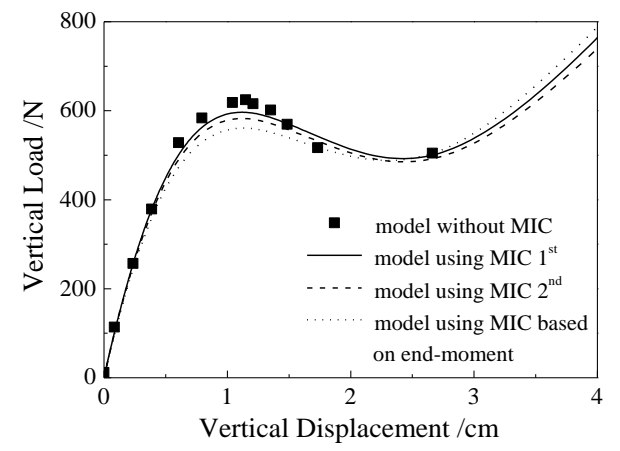

(a)Load-displacement curves with rigid joints

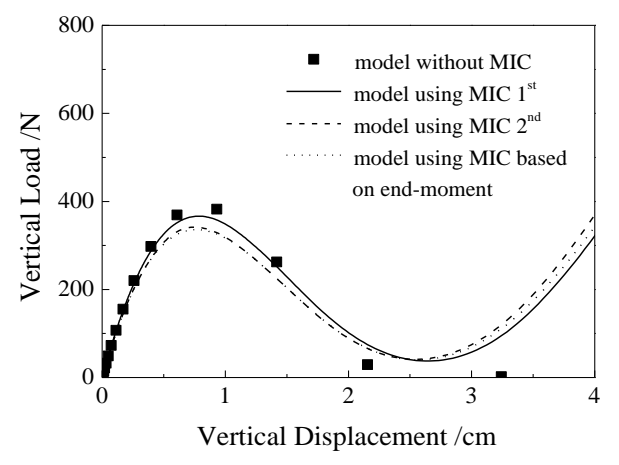

(b) Load-displacement curves with semi-rigid joints

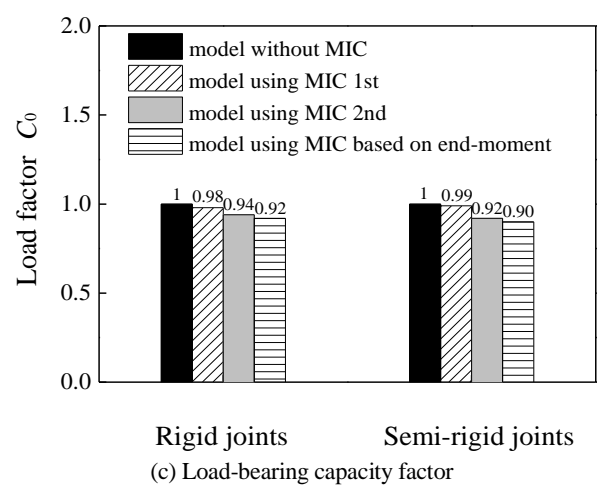

Fig. 13 Model results for the hexagon star dome

Fig. 13 (a) shows that the stability bearing capacities of the four types of rigid joints models are $617.2,601.9,577.1$, and $572.6 \mathrm{~N}$, and the capacity of the model using the type of member initial curvature based on the bars' end moments is the lowest. Fig. 13 (b) shows that the stability bearing capacities of the four types of semi-rigid joints models are 382.2, 377.1, 351.0, and $342.5 \mathrm{~N}$, and the capacity of the model using the type of member initial curvature based on the bars' end moments is also the lowest. These results are obtained because, in this system, the end moments of the compression bars around the middle loading point are opposite, and the supporting end moments of the compression bars around the supporting points are zero. The deformation shapes of the compression bars may present synclastic curvature or anticlastic curvature accordingly under load, proving that the model featuring different member initial curvatures based on the bars' end moments represents the most unfavourable condition for this dome system.

The analyses of the above mentioned systems verify that the stability loadbearing capacity of structures decreases if the model established considers member initial curvature. The extent to which the stability load-bearing capacity of the models considering different member initial curvatures decreases varies. The model established with member initial curvatures considering the bars' end moments represents the most unfavourable condition, and the extent to which the stability load-bearing capacity decreases in this case is the lowest. Moreover, the degrees to which the rigid or semi-rigid joints decrease the structural stability capacity are nearly the same, because the joint stiffness differences between the rigid and semi-rigid joints do not affect the direction of the end moments or the selected type of MIC functions.

\section{Application to single-layer spherical reticulated domes}

Through a full-range nonlinear analysis of single-layer spherical reticulated dome under a full-span vertical load, the influence of the structure stability loadbearing capacity considering different member initial curvatures is discussed. The analytical models of single-layer reticulated dome shown in Fig. 14 are employed in this study, including Kiewitt- 8 dome. The analytical parameters are the span $(L)$ and the rise-to-span ratio $(f / L)$, as listed in Table 1. For each dome, three types of dome models considering different member initial curvatures are established for comparison with models with no member initial curvatures. For each model, five types of member initial curvature amplitudes $\left(\delta_{\max } l l\right)$ are selected, as shown in Table 1 . The boundary conditions include fixed hinge support, and the material is Q235 steel, with a yield strength of $235 \mathrm{MPa}$ and an elastic modulus of $2.06 \times 10^{5} \mathrm{MPa}$. The steel stress-strain model of the single-layer reticulated dome is a combined-hardening model that possesses the characteristics of both isotropic and kinematic hardening models, as shown in Fig. 15. The material parameters of the steel constitutive model are obtained from experimental results [25].
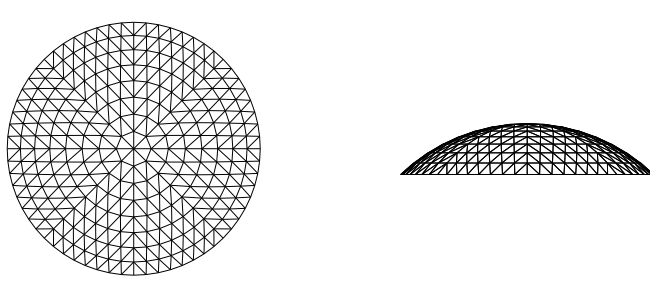

(a) Structure plan

(b) Structure elevation

Fig. 14 Analytical models of the single-layer reticulated dome 


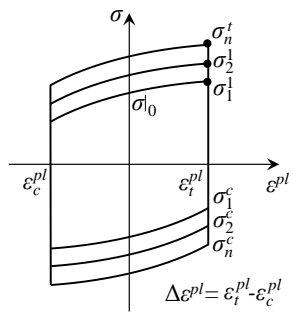

(a) Isotropic hardening

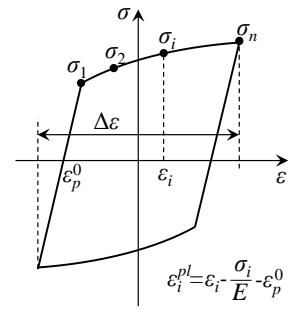

(b) Kinematic hardening
Fig. 15 Steel stress-strain curves

Table 1

Example of a table Parameters of single-layer reticulated domes

\begin{tabular}{cccccc}
\hline $\begin{array}{c}\text { Span } \\
L / m\end{array}$ & $\begin{array}{c}\text { Rise-to- } \\
\text { span ratio } \\
f / L\end{array}$ & $\begin{array}{c}\text { Numbers } \\
\text { of circles }\end{array}$ & $\begin{array}{c}\text { Radial and } \\
\text { circular bars }\end{array}$ & Diagonal bars & $\begin{array}{c}\text { MIC } \\
\text { amplitude } \delta / l\end{array}$ \\
\hline 40 & & 6 & $\Phi 133 \times 4$ & $\Phi 114 \times 3$ & $1 / 1000,1 / 500$, \\
60 & $1 / 4,1 / 5,1 / 6$ & 8 & $\Phi 152 \times 5.5$ & $\Phi 140 \times 5$ & $1 / 300,1 / 200$, \\
80 & & 10 & $\Phi 203 \times 6$ & $\Phi 194 \times 6$ & $1 / 100$ \\
\hline
\end{tabular}

The three types of dome models using member initial curvatures are MIC 1st only, MIC 2nd only, and different functions based on the bars' end moments. The load factors of the stability load-bearing capacity $C_{0}$ of the different dome models using different types of member initial curvatures with the same amplitude of $\delta_{\max }=l / 500$ are shown in Fig. 16.

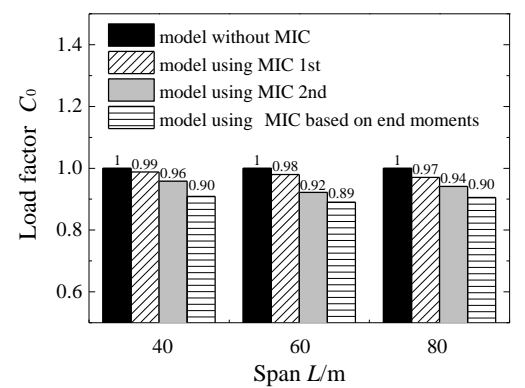

(a) Different spans

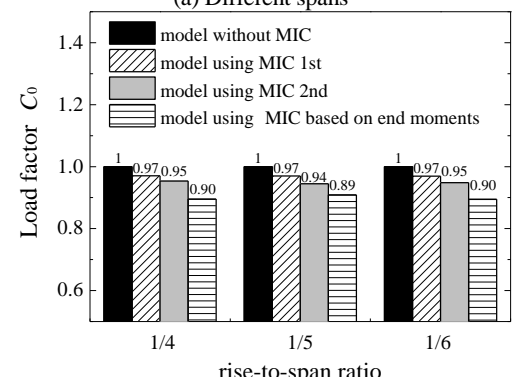

(b) Different rise-to-span ratios

Fig.16 Model results for single-layer reticulated dome

Fig. 16 verifies that the stability load-bearing capacity of the dome decreases if the model is established considering member initial curvature. The degree to which the stability load-bearing capacity of models considering different member initial curvatures decreases varies. The model established with member initial curvatures considering the bars' end moments represents the most unfavourable condition, and the degree to which its stability loadbearing capacity decreases is the lowest. The average load reduction factor $C_{0}$ of the different models using member initial curvatures considering end moments with the same amplitude of $\delta_{\max }=1 / 500$ is 0.90 . This result demonstrates that neither a change in span nor a change in the rise-to-span ratio has a significant influence on $C_{0}$ for the different types of models using the same amplitude of member initial curvatures considering end moments.

The reduction factors of the stability load-bearing capacity $C_{0}$ of the different dome models using different amplitudes of the member initial curvatures are shown in Fig. 17. Fig. 17 shows that an increase in the amplitude of the member initial curvatures results in a decrease in $C_{0}$. The average $C_{0}$ of the models with different spans or rise-to-span ratios using the same member initial curvature amplitudes of $l / 1000, l / 500, l / 300, l / 200$ and $l / 100$ are 0.96 , $0.91,0.85,0.81$ and 0.72 , respectively.

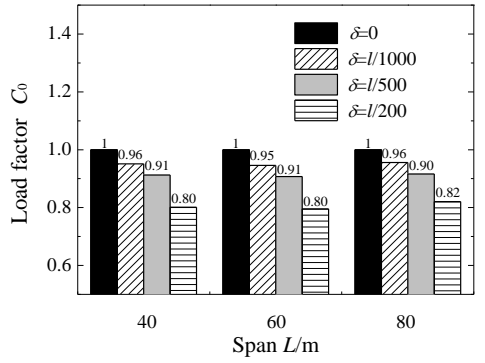

(a) Different spans

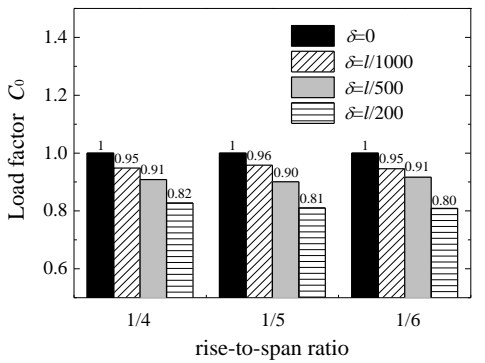

(b) Different rise-to-span ratios

Fig. 17 Amplitude results for single-layer reticulated dome

To choose a reasonable amplitude for the member initial curvature considering end moments, the relationships between the member initial curvature amplitudes and the load factor $C_{0}$ are plotted in Fig. 18 with the relationships between the member initial curvature amplitudes and the maximum displacements $U_{\max }$ in the dome' elastic ultimate state. Fig. 18 shows that the stability load-bearing capacity of the single-layer reticulated dome decreases linearly with increasing MIC amplitude. The maximum displacement $U_{\max }$ in the elastic limit state of the single-layer reticulated dome decreases precipitously when the member initial curvature amplitude is exceeds $l / 500$, this result suggests that a critical state is reached by the single-layer reticulated dome when the member initial curvature amplitude equals $/ / 500$. It is observed that the amplitude of MIC proposed in this paper is equal to that of Section Type A in the Hong Kong Steel Code [19] when adopting a hot-finished pipe section and greater than the value prescribed by the Chinese code [26], as shown in Table 2. These discrepancies occur because member residual stress and member initial eccentricity must be considered when the amplitude of member imperfection at $l / 1000$ does not meet the specified requirements [20]. Considering the internal force state of the structure and the existing code specifications, a member initial imperfection value of $l / 500$ is applicable for hotfinished structural hollow sections, which are commonly used in single-layer reticulated dome due to the equivalent biaxial cross-section stiffness, such as that of the pipe and box sections. Other types, such as $\mathrm{H}$ and I sections, which are seldom used in these domes due to the large difference of bending stiffness about the strong and weak cross-section axes, are not considered in this paper.

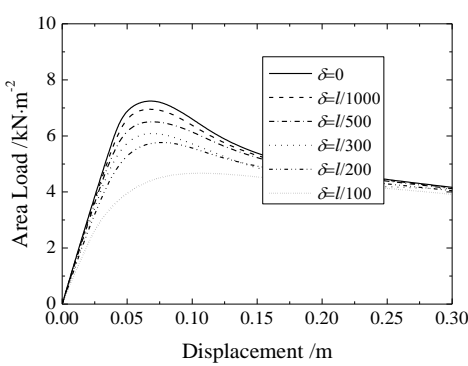

(a) Load-displacement curves

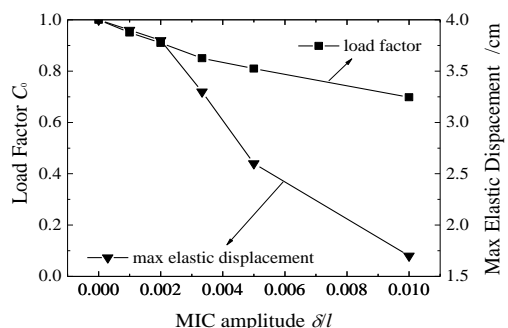

(b) Maximum elastic displacement 


\section{Conclusions}

(1) The stability bearing capacity of single-layer reticulated dome decreases when the associated numerical model considers the influence of the member initial curvature of each bar. The influence of the end moments transmitted by rigid joints or semi-rigid joints should be considered when numerical models of compression members are established using member initial curvatures.

Table 2

MIC amplitude of single-layer reticulated domes

\begin{tabular}{ccc}
\hline Representative specification & Type section & Amplitude $\delta_{\max } / l$ \\
\hline Eurocode3 & & $1 / 300$ \\
Hong Kong code & Hot-finished & $1 / 500$ \\
Chinese code & hollow section & $1 / 1000$ \\
Value recommended in this paper & & $1 / 500$ \\
\hline
\end{tabular}

(2) The most unfavourable condition occurs when the member initial curvature of the compression bar corresponds to the deformation shape at the critical state. There are two types of deflection shapes of compression bars considering end moments: one in which the signs of the moments at the two ends are the same and the other in which the signs are opposite. According to the two types of deflection curves, two forms of member initial curvatures are proposed: synclastic curvature and anticlastic curvature.

(3) Using the ABAQUS finite-element program, each bar of a single-layer reticulated dome is meshed using B31 elements by the multi-beam method to establish new analytical models considering member initial curvatures based on end moments. The approach performs a full-range nonlinear analysis of a perfect structure without any member defects first to obtain the moments of each bar at both ends in the critical state. Then, the coordinates of the internal nodes in each bar are modified by choosing different types of member initial curvatures depending on the bar's end moments and the bar defamation direction to obtain a new numerical model.

(4) The reduction degree of the stability load-bearing capacity of singlelayer reticulated dome when modelling using member initial curvatures based on end moments is greater than that of functions that do not consider end moments. The influence of the same amplitude of the member initial curvatures considering end moments is small when the single-layer reticulated dome' span and rise-to-span ratio vary. Varying the amplitude of the member initial curvatures considering end moments has a strong effect on the stability loadbearing capacity of single-layer reticulated dome. Based on the response of the structure and realistic construction conditions, $l / 500$ is proposed as a reasonable maximum value of the member initial curvature for hot-finished structural hollow sections.

\section{Acknowledgements}

This work was sponsored by the National Key Research and Development Program of China (Grant NO. 2016YFC0701103), the Major Research Plan of the National Natural Science Foundation of China (Grant NO. 91315301-06) and the Natural Science Foundation of Tianjin (Grant NO. 13JCZDJC35200). The financial support from the open project fund of the Key Laboratory of Coast Civil Structural Safety of the Ministry of Education of Tianjin University was also greatly appreciated.

\section{References}

[1] Chen X. and Shen S.Z., "Complete load-deflection response and initial imperfection analysis of single-layer lattice dome", International Journal of Space Structures, 1993, 8(4), 271-278.

[2] Gioncu V., "Buckling of reticulated shells: state-of-the-art." International Journal of Space Structures, 1995, 10(1), 1-46

[3] BSI, "EN 1993-1-6. Eurocode 3: Design of steel structures-Part 1-6: Strength and stability of shell structures", European Committee for Standardization: Brussels, 2007.

[4] Mohurd "JGJ 7-2010: Technical specification for space frame structures", Beijing: China Architecture Industry Press, 2010. (in Chinese)

[5] Adman R. and Afra H., "Exact shape functions of imperfect beam element for stability analysis", Advances in Engineering Software, 2007, 38(8), 576-585

[6] Li G.Q. and Liu Y.S.,"A nonlinear beam element considering initial imperfection", Chinese Journal of Computational Mechanics, 2005, 22(1), 69-72. (in Chinese)

[7] Kumar P.A., Sahoo D.R. and Kumar N., "Limiting values of slenderness ratio for circular braces of concentrically braced frames", Journal of Constructional Steel Research 2015, 115, 223-235.

[8] Lotfollahi M., Alinia M.M. and Taciroglu E., "Validated finite element techniques for quasistatic cyclic response analyses of braced frames at sub-member scales", Engineering Structures, 2016, 106, 222-242.

[9] Liew J.R., Punniyakotty N.M. and Shanmugam N.E., "Advanced analysis and design of spatial structures", Journal of Constructional Steel Research, 1997, 42(1), 21-48.

[10] Qi L., Shao Y. and Huang Z., et al, "Dynamic damage criterion and damage mode for single layer lattice shell", Journal of Constructional Steel Research, 2014, 99, 102-110.

[11] Marshall P.W., Gates W.E. and Anagnostopoulos S.W., "Inelastic dynamic analysis of tubular offshore structures", Proceedings of Ninth Annual Offshore Technology Conference Offshore Technology Conference, Houston, USA, 1977, 235-246.

[12] Ding Y., Chen Z.T. and Zong L., et al, "A theoretical strut model for severe seismic analysis of single-layer reticulated dome", Journal of Constructional Steel Research, 2017, 128, 661671.

[13] Chan S.L. and Zhou Z.H., "Second order analysis of frame using a single imperfect element per member", Journal of Structural Engineering, ASCE, 1995, 121(6), 939-945.

[14] Fan F., Yan J. and Cao Z., "Stability of reticulated shells considering member buckling", Journal of Constructional Steel Research, 2012, 77, 32-42.

[15] Yan J.C., Qin F. and Cao Z., et al, "Mechanism of coupled instability of single-layer reticulated dome", Engineering Structures, 2016, 114, 158-170.

[16] Zhou Z., Wu J. and Meng S.P., "Nonlinear stability bearing capacity analysis for a suspended dome based on the initial curvature elements", Chinese Journal of Computational Mechanics, 2010, 27(4), 721-726. (in Chinese)

[17] Yan J., Fan F. and Cao Z., "Research on influence of initial curvature of members on elastoplastic stability of reticulated shells", Journal of Building Structures, 2012, 33(12), 63-71. (in Chinese)

[18] Wang Q., Deng H. and Huang L., "Effect of member's initial curvature on the static and dynamic bearing capacity of suspended dome", Spatial Structures, 2013, 19(4), 18-24. (in Chinese)

[19] Code of Practice for the Structural Use of Steel 2011., Buildings Department of the Government of the Hong Kong Special Administrative Region, Hong Kong, China, 2011.

[20] Pang P.T.C., Kwan K.K., and Chan S.L., "Hong Kong code of practice for the structural use of steel 2005-second-order analysis and design method with effective-length-free assumption", Progress in Steel Building Structures, 2007, 9(5), 57-62. (in Chinese)

[21] Chan S.L., "Geometric and material non-linear analysis of beam-columns and frames using the minimum residual displacement method", International Journal for Numerical Methods in Engineering, 1988, 26(12), 2657-2669.

[22] Shen S.Z. and Chen X., "Stability of reticulated shells", Beijing: Science Press, 1999, 6970. (in Chinese).

[23] Papadrakakis M., "Post-buckling analysis of spatial structures by vector iteration methods", Computers \& structures, 1981, 14(5-6), 393-402.

[24] Meek J.L. and Tan H.S., "Geometrically nonlinear analysis of space frames by an incremental iterative technique", Computer methods in applied mechanics and engineering, 1984, 47(3), 261-282.

[25] Shi Y.J., Meng W., Wang Y.Q., "Experimental study of structural steel constitutive relationship under cyclic loading", Journal of Building Materials, 2012, 15(3), 293-300. (in Chinese)

[26] Ministry of Construction of the People's Republic of China, "GB 50017-2003: Code of design of steel structures", Beijing: China Planning Press, 2003. (in Chinese). 